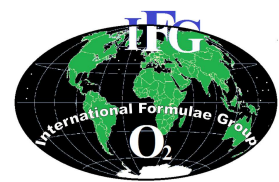

Available online at http://ajol.info/index.php/ijbes

Int. J. Biol. Chem. Sci. 9(1): 217-224, February 2015

International Journal

of Biological and

Chemical Sciences

ISSN 1997-342X (Online), ISSN 1991-8631 (Print)

Original Paper

http://indexmedicus.afro.who.int

\title{
Comportement de principaux bioagresseurs et maladies du cotonnier sur les variétés éprouvées de cotonnier (Gossypium hirsutum) sous différentes doses d'engrais à Angaradébou au Bénin
}

\author{
Thomas Aïdjo HOUNDETE ${ }^{1 *}$, Alexis HOUGNI ${ }^{1}$, Saliou ALADJI ${ }^{2}$, Augustin DAGOUDO ${ }^{2}$ \\ Nouhoun ZOUMAROU-WALLIS ${ }^{2}$ et Agnès A. THOMAS-ODJO ${ }^{2}$ \\ ${ }^{I}$ Centre de Recherches Agricoles Coton et Fibres, Institut National des Recherches Agricoles du Bénin, \\ 01 BP 175, Cotonou, Bénin. \\ ${ }^{2}$ Faculté d'Agronomie, Université de Parakou, BP 123, Parakou, Bénin. \\ *Auteur correspondant; E-mail:houndetet@yahoo.fr; 01 BP 175 Cotonou, Bénin, \\ Tél. +22995810321/96044416
}

\section{RESUME}

Au Bénin, la filière coton participe activement à l'économie nationale.Quatre nouvelles variétés issues du Centre de Recherches Agricoles Coton et Fibres ont été testées au Nord Bénin dans l'optique d'identifier la variété la mieux adaptée. Ces variétés ont été comparées à la variété H 279-1en vulgarisation. L'objectif est d'évaluer les comportements des variétés vis-à-vis des bioagresseurs et des maladies dans la zone. Le dispositif expérimental est un split plot à six répétitions, composé d'un facteur principal représenté par trois doses d'engrais chimique à savoir D1 (témoin) $=150 \mathrm{~kg}$ de NPKSB $+50 \mathrm{~kg}$ d'Urée à l'hectare, D2=200 kg de $\mathrm{NPKSB}+50 \mathrm{~kg}$ d'Urée à l'hectare et D3= $250 \mathrm{~kg}$ de NPKSB + $50 \mathrm{~kg}$ d'Urée à l'hectare. Le facteur secondaire est composé de cinq variétés : H 279-1, I875-3, E956-2, H769-5 et H782-3. Il a été révélé que la pression parasitaire a été la même au niveau de toutes les variétés et ne dépend pas des doses d'engrais appliquées. Les variétés E956-2 et H782-3 sont moins sensibles à la bactériose que les variétés H 279-1, I875-3 et H 769-5 avec des valeurs respectivement de 0,$37 ; 0,29 ; 0,67 ; 0,68$ et 0,59 plants attaqués sur 10 observés. (c) 2015 International Formulae Group. All rights reserved.

Mots clés: Comportement, variétés, engrais chimique, pression parasitaire, ravageurs, bactériose.

\section{INTRODUCTION}

Le cotonnier (Gossypium hirsutum), est la première plante textile cultivée au monde et constitue plus de $50 \%$ du marché des fibres (Celini, 2001). Le cotonnier est également une plante vivrière grâce aux graines de coton «glandless» (Lançon, 1996; Tchatchueng et Porte, 2008) et représente la deuxième source mondiale de protéine végétale avec une production de 12 millions de tonnes de tourteaux par an (Estur et Raymond, 1988).

Au Bénin, la filière coton est un des principaux moteurs de l'économie nationale à l'instar d'un certain nombre de pays de l'Afrique Zone franc (AZF) notamment le Mali, le Burkina Faso, le Tchad et le Togo (Hussein et al., 2005; Hougni, 2009). Plus de 325000 producteurs cultivent le coton faisant 
vivre environ 2 millions d'individus (Kpadé, 2011). La production est plus concentrée dans la partie nord du pays où elle occupe plus de $80 \%$ des paysans. Le coton reste alors la principale culture d'exportation qui contribue au développement socio-économique du Bénin avec 70 à $80 \%$ des recettes d'exportation, $35 \%$ des rentrées fiscales et une contribution en termes de valeur ajoutée estimée à 13\% du PIB (Dègla, 2012).

L'une des composantes de la culture cotonnière susceptible d'amélioration pour une meilleure rentabilité de la filière est la qualité de coton graine, grâce à l'introduction de nouvelles variétés. Les nouvelles variétés ainsi introduites permettent de diminuer l'impact des contraintes biotiques (ravageurs, maladies) et abiotiques (pauvreté, acidité et salinité des sols, sécheresse). Les priorités à retenir pour le Bénin au niveau de cette composante doivent absolument tenir compte des caractéristiques des systèmes de production que l'on rencontre dans le pays : l'essentiel du coton y est produit dans des exploitations de petites tailles avec un faible apport d'engrais et de pesticides alors que les niveaux de contraintes biotiques et abiotiques existants y sont élevés. Dans ce contexte, les réponses prioritaires à apporter par le programme d'amélioration variétale aux contraintes existantes au Bénin devraient viser le développement des variétés à fibres de haute qualité, multi-résistantes aux adversités biotiques et abiotiques (Guy, 2006). C'est ce que la présente recherche tente de faire en intégrant dans la sélection de nouvelles variétés régionalisées, l'étude de leurs comportements face aux ravageurs majeurs et aux maladies potentielles.

\section{MATERIEL ET METHODES Matériel végétal et chimique}

Les variétés I 875-3, E 956-2, H 769-5

et $\mathrm{H}$ 782-3 mises au point au Bénin ont été comparées à la variété H 279-1 actuellement en vulgarisation à Angaradébou en 2011-2012 et 2012-2013. Les parcelles ont été fumées aux engrais urée (46\%) et NPKSB (14-23-1451) à différentes doses. La protection phytosanitaire a été assurée avec des insecticides tels que Tihan175 O-Teq (Flubendiamide 100-spirotétramate 75), EmaSuper 56 DC (Emamectine 24-acetamipride 32) et Thunder 145 O-Teq (Bêtacyfluthrine 45-imidaclopride 100).

\section{Dispositif expérimental}

Le dispositif expérimental est un split plot à 3 objets principaux (doses d'engrais) et 5 objets secondaires (variétés) avec des parcelles secondaires de 5 lignes de $9 \mathrm{~m}$ chacune répétées 6 fois. Les parcelles sont semées à cinq (5) graines par poquet à écartements de $0,80 \mathrm{~m}$ entre lignes et $0,30 \mathrm{~m}$ entre les poquets avec un démariage à un plant par poquet.

\section{Application de fumure}

Pour cette expérimentation, on a apporté les doses de 150, 200 et $250 \mathrm{~kg} / \mathrm{ha}$ de NPKSB suivant les traitements à 20 jours après semis et $50 \mathrm{~kg} /$ ha d'urée à 45 jours après semis.

Ainsi, de facon spécifique, on a apporté $240 \mathrm{~g}, 320 \mathrm{~g}$ et $400 \mathrm{~g}$ de NPKSB et $80 \mathrm{~g}$ d'urée par ligne de $20 \mathrm{~m}$. L'apport est fait en ligne, et à $5 \mathrm{~cm}$ des poquets.

\section{Protection phytosanitaire}

Le contrôle des ravageurs du cotonnier a suivi le programme de traitement calendaire vulgarisé au Bénin. Au total, six (6) traitements ont été effectués durant la campagne à 14 jours d'intervalle à partir de $45^{\text {ème }}$ jour après la levée. Les observations sont réalisées la veille de chaque traitement insecticide.

Evaluation du comportement des variétés vis-à-vis des insectes ravageurs et des maladies

Le comptage hebdomadaire des insectes ravageurs a démarré à 44 jours après la levée. Cette opération a consisté à dénombrer sur 10 plants de la ligne 5 de chaque parcelle, Helicoverpa armigera, Diparopsis watersi et Dysdercus völkeri, et le nombre de plants attaqués par la mouche 
blanche Bemisia tabaci et les pucerons Aphis gossypii. La bactériose a été aussi observée dans les mêmes conditions en comptant le nombre de plants atteints.

Enfin, la récolte a été faite sur les 3 lignes centrales de chacune des parcelles élémentaires sur une superficie de 21,6 m².

\section{Analyse statistique des données}

L'analyse statistique des données collectées sur 2 ans a été réalisée à l'aide du logiciel SPSS version 18. Le test de StudentNewman-Keuls (SNK) a été utilisé pour séparer les variétés et les doses d'engrais en des groupes homogènes.

\section{RESULTATS}

Attaque des variétés par Helicoverpa armigera

Le nombre moyen de Helicoverpa armigera dénombré sur 10 plants durant la période d'observation varie entre 1,33 $( \pm 0,11)$ pour la variété I $875-3$ et $1,501( \pm 0,11)$ pour la variété $H$ 279-1. L'analyse de variance des données ne révèle aucune différence significative ni entre les variétés, ni les doses d'engrais et ni l'interaction (dose $\mathrm{x}$ variété) au seuil de $5 \%$. Le nombre de $H$. armigera observé sur 10 plants ne varie donc pas d'une variété à l'autre et n'est pas fonction des doses d'engrais appliquées (Tableau 1). H. armigera n'a pas une attraction particulière vis-à-vis des variétés. Il n'y a donc ni effet variété, ni effet dose d'engrais dans le comportement de $H$. armigera en culture cotonnière à Angaradébou.

\section{Attaque des variétés par Diparopsis watersi}

Le nombre moyen de Diparopsis watersi dénombré sur 10 plants varie entre $0,03( \pm 0,01)$ pour $\mathrm{H} 782-3$ et $0,05( \pm 0,01)$ pour $\mathrm{H}$ 279-1. L'analyse de variance des données au niveau des plants attaqués par $D$. watersi, ne révèle aucune différence significative entre les variétés, les doses et l'interaction (dose $\mathrm{x}$ variété) au seuil de 5\% est inexistante (Tableau 2). D. watersi n'a ni de préférence variétale, ni celle des doses d'engrais en culture cotonnière à Angaradébou.

\section{Attaque des variétés par pucerons Aphis gossypii \\ Le nombre moyen de plants infestés par} les pucerons sur 10 plants observés varie entre 0,07 $( \pm 0,04)$ pour la variété H 769-5 et 0,23 ( $\pm 0,04)$ pour la variété I 875-3 (Tableau 3). L'analyse de variance des données révèle qu'il existe une différence significative entre les variétés par rapport à leur sensibilité vis-àvis de la pression des pucerons. La variété I 875-3 ayant montré une sensibilité statistiquement plus forte que les autres. La variété H 769-5 s'est révélée moins sensibles aux attaques de l'espèce Aphis gossypii. La dose sur laquelle on observe moins d'attaque est la dose $150 \mathrm{~kg} / \mathrm{ha}$ de NPKSB.

\section{Attaque des variétés par mouche blanche Bemisia tabaci}

Le nombre moyen de plants infestés par la mouche blanche Bemisia tabaci, dénombré sur 10 plants varie entre $0,03( \pm 0,02)$ pour la variété $H 769-5$ et $0,08( \pm 0,02)$ pour la variété H 782-3 (Tableau 4). L'analyse de variance des données indique qu'il n'existe aucune différence significative entre la sensibilité des variétés vis-à-vis de la pression des mouches blanches. B. tabaci, n'a donc pas de préférence variétale en culture cotonnière à Angaradébou.

\section{Attaque des variétés par Dysdercus völkeri \\ Le nombre moyen de Dysdercus völkeri dénombré sur 10 plants varie entre $0,03( \pm 0,02)$ pour $\mathrm{H} 769-5$ et $0,06( \pm 0,02)$ pour H 279-1. L'analyse de variance des données au niveau des plants attaqués, ne révèle aucune différence significative entre les variétés, les doses et l'interaction (dose*variété) au seuil de 5\% (Tableau 5). D. völkeri, n'a donc pas de préférence variétale en culture cotonnière à Angaradébou.}

\section{Attaque des variétés par la bactériose}

Le nombre moyen de plants infectés de bactériose dénombré sur 10 plants varie entre 
$0,29( \pm 0,07)$ pour la variété $H 782-3$ et 0,68 $( \pm 0,07)$ pour la variété $H$ 279-1 (Tableau 6).

L'analyse de variance met en évidence des différences hautement significatives $(\mathrm{p}<0,01)$ aussi bien au niveau des variétés qu'au niveau des doses d'engrais appliquées. A cet effet, il apparaît que les variétés E 956-2 et H 782-3 sont moins sensibles à la bactériose par rapport à H 279-1, I 875-3 et H 769-5. On peut également noter que les variétés ayant reçu de fortes doses d'engrais sont moins attaquées que celles qui ont reçu de faibles doses.

\section{Rendement moyen de coton graine}

Le rendement moyen de coton graine varie entre $2769,9( \pm 289,8) \mathrm{kg} / \mathrm{ha}$ pour $\mathrm{H}$
782-3 et 3098,6 $( \pm 298,8) \mathrm{kg} / \mathrm{ha}$ pour E $956-2$. L'analyse de variance des rendements toutes doses confondues révèle une différence significative au seuil de 5\% entre les variétés. Trois (3) groupes homogènes ont été discriminés avec la variété E 956-2 en tête, $H$ 782-3 en dernier et I 875-3; H 769-5 et H 2791 dans le groupe intermédiaire. Toutefois, une différence hautement significative s'observe lorsqu'on considère les doses d'engrais, toutes variétés confondues. Ceci indique que le niveau de rendement a été fonction du niveau de la dose appliquée au seuil de 1\% (Tableau 7).

Tableau 1: Nombre moyen de Helicoverpa armigera sur 10 plants en fonction des variétés et des doses.

\begin{tabular}{lcccccccc}
\hline Variétés & D1 & $\mathbf{\pm}$ ES & D2 & $\mathbf{E ~ E S}$ & D3 & $\mathbf{E S S}$ & Moy & $\mathbf{\pm}$ ES \\
\hline H 279-1 & 1,44 & $\pm 0,18$ & 1,81 & $\pm 0,18$ & 1,26 & $\pm 0,18$ & 1,50 & $\pm 0,11$ \\
I 875-3 & 1,37 & $\pm 0,18$ & 1,32 & $\pm 0,18$ & 1,29 & $\pm 0,18$ & 1,33 & $\pm 0,11$ \\
E 956-2 & 1,50 & $\pm 0,18$ & 1,46 & $\pm 0,18$ & 1,54 & $\pm 0,18$ & 1,50 & $\pm 0,11$ \\
H 769-5 & 1,46 & $\pm 0,18$ & 1,46 & $\pm 0,18$ & 1,28 & $\pm 0,18$ & 1,39 & $\pm 0,11$ \\
H 782-3 & 1,40 & $\pm 0,18$ & 1,19 & $\pm 0,18$ & 1,48 & $\pm 0,18$ & 1,36 & $\pm 0,11$ \\
Moy & 1,44 & $\pm 0,08$ & 1,45 & $\pm 0,08$ & 1,37 & $\pm 0,08$ & & \\
\hline & Variété & Dose & Dose X var & & & & & \\
P (5\%) & $0,685 \mathrm{~ns}$ & 0,786ns & 0,630ns & & & & &
\end{tabular}

D1: Dose de $150 \mathrm{~kg}$ de NPKSB+ $50 \mathrm{~kg}$ d'Urée /ha, D2: Dose de $200 \mathrm{~kg}$ de NPKSB+ $50 \mathrm{~kg}$ d'Urée /ha, D3: Dose de $250 \mathrm{~kg}$ de NPKSB+ $50 \mathrm{~kg}$ d'Urée /ha; ES: Erreur Standard; Moy: Moyenne ; P: Probabilité, ns: effet non significatif.

Tableau 2: Nombre de Diparopsis watersi sur 10 plants en fonction des variétés et des doses.

\begin{tabular}{|c|c|c|c|c|c|c|c|c|}
\hline Variétés & D1 & $\pm \mathrm{ES}$ & D2 & $\pm \mathrm{ES}$ & D3 & $\pm \mathrm{ES}$ & Moy & $\pm \mathrm{ES}$ \\
\hline H 279-1 & 0,06 & $\pm 0,02$ & 0,03 & $\pm 0,02$ & 0,06 & $\pm 0,02$ & 0,05 & $\pm 0,01$ \\
\hline I $875-3$ & 0,00 & $\pm 0,02$ & 0,07 & $\pm 0,02$ & 0,04 & $\pm 0,02$ & 0,04 & $\pm 0,01$ \\
\hline E 956-2 & 0,04 & $\pm 0,02$ & 0,01 & $\pm 0,02$ & 0,04 & $\pm 0,02$ & 0,03 & $\pm 0,01$ \\
\hline Н 769-5 & 0,03 & $\pm 0,02$ & 0,04 & $\pm 0,02$ & 0,03 & $\pm 0,02$ & 0,03 & $\pm 0,01$ \\
\hline H 782-3 & 0,04 & $\pm 0,02$ & 0,03 & $\pm 0,02$ & 0,03 & $\pm 0,02$ & 0,03 & $\pm 0,01$ \\
\hline Moy & 0,03 & $\pm 0,01$ & 0,04 & $\pm 0,01$ & 0,04 & $\pm 0,01$ & & \\
\hline$P(5 \%)$ & $\begin{array}{c}\text { Variété } \\
0,942 \mathrm{~ns}\end{array}$ & $\begin{array}{c}\text { Dose } \\
0,934 \mathrm{~ns}\end{array}$ & $\begin{array}{c}\text { Dose } \mathrm{x} \text { var } \\
0,595 \mathrm{~ns}\end{array}$ & & & & & \\
\hline
\end{tabular}


Tableau 3: Nombre de plants infesté par les pucerons sur 10 plants en fonction des variétés et des doses

\begin{tabular}{lcccccccc}
\hline Variétés & D1 & $\mathbf{\pm E S}$ & $\mathbf{D 2}$ & $\mathbf{\pm E S}$ & $\mathbf{D 3}$ & $\mathbf{\pm E S}$ & Moy & $\mathbf{\pm E S}$ \\
\hline H 279-1 & 0,22 & $\pm 0,07$ & 0,15 & $\pm 0,07$ & 0,06 & $\pm 0,07$ & $0,14 \mathrm{ab}$ & $\pm 0,04$ \\
I 875-3 & 0,22 & $\pm 0,07$ & 0,25 & $\pm 0,07$ & 0,22 & $\pm 0,07$ & $0,23 \mathrm{~b}$ & $\pm 0,04$ \\
E 956-2 & 0,17 & $\pm 0,07$ & 0,07 & $\pm 0,07$ & 0,37 & $\pm 0,07$ & $0,20 \mathrm{ab}$ & $\pm 0,04$ \\
H 769-5 & 0,04 & $\pm 0,07$ & 0,07 & $\pm 0,07$ & 0,11 & $\pm 0,07$ & $0,07 \mathrm{a}$ & $\pm 0,04$ \\
H 782-3 & 0,15 & $\pm 0,07$ & 0,29 & $\pm 0,07$ & 0,11 & $\pm 0,07$ & $0,19 \mathrm{ab}$ & $\pm 0,04$ \\
Moy & 0,16 & $\pm 0,03$ & 0,17 & $\pm 0,03$ & 0,17 & $\pm 0,03$ & & \\
\hline & Variété & Dose & Dose x var & & & & & \\
P (5\%) & $0,040 \mathrm{~s}$ & $0,947 \mathrm{~ns}$ & $0,018 \mathrm{~s}$ & & & & & \\
\hline
\end{tabular}

D1: Dose de $150 \mathrm{~kg}$ de NPKSB+ $50 \mathrm{~kg}$ d'Urée /ha, D2: Dose de $200 \mathrm{~kg}$ de NPKSB+ $50 \mathrm{~kg}$ d'Urée /ha, D3: Dose de $250 \mathrm{~kg}$ de NPKSB+ $50 \mathrm{~kg}$ d'Urée /ha; ES: Erreur Standard; Moy: Moyenne; P: Probabilité, s: effet significatif, ns: effet non significatif. Les moyennes affectées de différentes lettres indiquent des différences significatives entre les traitements après le test de Student-Newman-Keuls $(\mathrm{P}<0,05)$, celles suivies de la même lettre ne sont pas significativement différentes $(\mathrm{P}>0,05)$ d'après le test.

Tableau 4 : Nombre de plants infestés de Bemisia tabaci sur 10 plants en fonction des variétés et des doses.

\begin{tabular}{lcccccccc}
\hline variétés & D1 & $\mathbf{\pm E S}$ & $\mathbf{D 2}$ & $\mathbf{\pm}$ ES & $\mathbf{D 3}$ & $\mathbf{E}$ ES & Moy & $\mathbf{\pm}$ ES \\
\hline H 279-1 & 0,06 & $\pm 0,03$ & 0,03 & $\pm 0,03$ & 0,06 & $\pm 0,03$ & 0,05 & $\pm 0,02$ \\
I 875-3 & 0,03 & $\pm 0,03$ & 0,04 & $\pm 0,03$ & 0,11 & $\pm 0,03$ & 0,06 & $\pm 0,02$ \\
E 956-2 & 0,01 & $\pm 0,03$ & 0,00 & $\pm 0,03$ & 0,07 & $\pm 0,03$ & 0,03 & $\pm 0,02$ \\
H 769-5 & 0,03 & $\pm 0,03$ & 0,06 & $\pm 0,03$ & 0,00 & $\pm 0,03$ & 0,03 & $\pm 0,02$ \\
H 782-3 & 0,15 & $\pm 0,03$ & 0,06 & $\pm 0,03$ & 0,03 & $\pm 0,03$ & 0,08 & $\pm 0,02$ \\
Moy & 0,06 & $\pm 0,014$ & 0,04 & $\pm 0,01$ & 0,05 & $\pm 0,01$ & & \\
\hline & Variété & Dose & Dose x Var & & & & & \\
P (5\%) & $0,24 n s$ & $0,588 n s$ & $0,049 n s$ & & & & & \\
\hline
\end{tabular}

D1: Dose de $150 \mathrm{~kg}$ de NPKSB+ $50 \mathrm{~kg}$ d'Urée /ha, D2: Dose de $200 \mathrm{~kg}$ de NPKSB+ $50 \mathrm{~kg}$ d'Urée /ha, D3: Dose de $250 \mathrm{~kg}$ de NPKSB+ $50 \mathrm{~kg}$ d'Urée /ha; ES: Erreur Standard; Moy: Moyenne ; P: Probabilité, ns: effet non significatif.

Tableau 5: Nombre de Dysdercus völkeri sur 10 plants en fonction des variétés et des doses

\begin{tabular}{lcccccccc}
\hline Variétés & D1 & $\mathbf{E}$ ES & D2 & $\mathbf{\pm E S}$ & $\mathbf{D 3}$ & $\mathbf{E}$ ES & Moy & $\pm \mathbf{E S}$ \\
\hline H 279-1 & 0,06 & $\pm 0,03$ & 0,06 & $\pm 0,03$ & 0,08 & $\pm 0,03$ & 0,06 & $\pm 0,02$ \\
I 875-3 & 0,00 & $\pm 0,03$ & 0,06 & $\pm 0,03$ & 0,04 & $\pm 0,03$ & 0,03 & $\pm 0,02$ \\
E 956-2 & 0,03 & $\pm 0,03$ & 0,03 & $\pm 0,03$ & 0,07 & $\pm 0,03$ & 0,04 & $\pm 0,02$ \\
H 769-5 & 0,06 & $\pm 0,03$ & 0,04 & $\pm 0,03$ & 0,00 & $\pm 0,03$ & 0,03 & $\pm 0,02$ \\
H 782-3 & 0,00 & $\pm 0,03$ & 0,13 & $\pm 0,03$ & 0,00 & $\pm 0,03$ & 0,04 & $\pm 0,02$ \\
Moy & 0,03 & $\pm 0,01$ & 0,06 & $\pm 0,01$ & 0,04 & $\pm 0,01$ & & \\
& Variété & Dose & Dose x Var & & & & & \\
P (5\%) & 0,716 ns & $0,238 \mathrm{~ns}$ & $0,130 \mathrm{~ns}$ & & & & & \\
\hline
\end{tabular}

D1: Dose de $150 \mathrm{~kg}$ de NPKSB+ $50 \mathrm{~kg}$ d'Urée /ha, D2: Dose de $200 \mathrm{~kg}$ de NPKSB+ $50 \mathrm{~kg}$ d'Urée /ha, D3: Dose de $250 \mathrm{~kg}$ de NPKSB+ $50 \mathrm{~kg}$ d'Urée /ha; ES: Erreur Standard; Moy: Moyenne ; P: Probabilité, ns: effet non significatif. 
Tableau 6: Nombre de plants atteints de bactériose sur 10 plants en fonction des variétés et des doses.

\begin{tabular}{|c|c|c|c|c|c|c|c|c|}
\hline Variétés & D1 & $\pm \mathbf{E S}$ & D2 & $\pm \mathbf{E S}$ & D3 & $\pm \mathbf{E S}$ & Moy & $\pm \mathbf{E S}$ \\
\hline H 279-1 & 0,90 & $\pm 0,13$ & 0,83 & $\pm 0,13$ & 0,27 & $\pm 0,13$ & $0,67 b$ & $\pm 0,07$ \\
\hline I 875-3 & 0,85 & $\pm 0,13$ & 0,69 & $\pm 0,13$ & 0,48 & $\pm 0,13$ & $0,68 b$ & $\pm 0,07$ \\
\hline E 956-2 & 0,50 & $\pm 0,13$ & 0,35 & $\pm 0,13$ & 0,26 & $\pm 0,13$ & $0,37 \mathrm{a}$ & $\pm 0,07$ \\
\hline H 769-5 & 0,85 & $\pm 0,13$ & 0,58 & $\pm 0,13$ & 0,36 & $\pm 0,13$ & $0,59 b$ & $\pm 0,07$ \\
\hline H 782-3 & 0,18 & $\pm 0,13$ & 0,40 & $\pm 0,13$ & 0,31 & $\pm 0,13$ & $0,29 \mathrm{a}$ & $\pm 0,07$ \\
\hline Moy & $0,66 \mathrm{~b}$ & $\pm 0,06$ & $0,57 b$ & $\pm 0,06$ & $0,34 \mathrm{a}$ & $\pm 0,06$ & & \\
\hline $\mathrm{P}(5 \%)$ & $\begin{array}{l}\text { Variété } \\
0,000 \text { hs }\end{array}$ & $\begin{array}{l}\text { Dose } \\
0,001 \mathrm{hs}\end{array}$ & $\begin{array}{l}\text { Dose } \mathrm{x} \text { var } \\
0,175 \mathrm{~ns} \\
\end{array}$ & & & & & \\
\hline
\end{tabular}

D1: Dose de $150 \mathrm{~kg}$ de NPKSB+ 50 kg d'Urée /ha, D2: Dose de $200 \mathrm{~kg}$ de NPKSB+ 50 kg d'Urée /ha, D3: Dose de 250 kg de NPKSB+ $50 \mathrm{~kg}$ d'Urée /ha ; ES: Erreur Standard; Moy: Moyenne ; P: Probabilité, hs: effet hautement significatif, ns: effet non significatif. Les moyennes affectées de différentes lettres indiquent des différences significatives entre les traitements après le test de Student-Newman-Keuls $(\mathrm{P}<0,05)$, celles suivies de la même lettre ne sont pas significativement différentes $(\mathrm{P}>0,05)$ d'après le test.

Tableau 7: Rendement de coton graine (kg/ha) par variété et par dose au CPE d'Angaradébou (moyenne \pm erreur standard).

\begin{tabular}{lccccccc}
\hline \multirow{2}{*}{ Variétés } & \multicolumn{7}{c}{ Doses } \\
\cline { 2 - 7 } & \multicolumn{2}{c}{ D1 \pm ES } & \multicolumn{2}{c}{ D2 \pm ES } & D3 \pm ES & Moy ( \pm ES) \\
\hline H 279-1 & 2684.0 & $\pm 424,0$ & 2999.0 & $\pm 424,0$ & 3089,5 & $\pm 176,5$ & $2924,2 \pm 341,5 \mathrm{ab}$ \\
I 875-3 & 2873.0 & $\pm 486,0$ & 2862,7 & $\pm 137,4$ & 3125,2 & $\pm 210,8$ & $2953,6 \pm 278,1 \mathrm{ab}$ \\
E 956-2 & 2922.0 & $\pm 335,0$ & 3029.0 & $\pm 346,0$ & 3344,8 & $\pm 215,5$ & $3098,6 \pm 298,8 \mathrm{a}$ \\
H 769-5 & 2748.0 & $\pm 329,0$ & 2912,8 & $\pm 116,9$ & 2990,8 & $\pm 181,9$ & $2883,9 \pm 209,3 \mathrm{ab}$ \\
H 782-3 & 2509,8 & $\pm 216,4$ & 2772,0 & $\pm 347,0$ & 3028.0 & $\pm 306,0$ & $2769,9 \pm 289,8 \mathrm{~b}$ \\
Moy & 2747,4 & $\pm 358,1 \mathrm{c}$ & 2915,1 & $\pm 274,3 \mathrm{~b}$ & 3115,7 & $\pm 218,1 \mathrm{a}$ & \\
\hline Source & var & dose & Var x dose & & & \\
P & $0,033 \mathrm{~s}$ & $0,000 \mathrm{hs}$ & $0,882 \mathrm{~ns}$ & & & & \\
\hline
\end{tabular}

D1: Dose de $150 \mathrm{~kg}$ de NPKSB+ $50 \mathrm{~kg}$ d'Urée /ha, D2: Dose de $200 \mathrm{~kg}$ de NPKSB+ $50 \mathrm{~kg}$ d'Urée /ha, D3: Dose de $250 \mathrm{~kg}$ de NPKSB+ $50 \mathrm{~kg}$ d'Urée /ha

ES: Erreur Standard; Moy: Moyenne

P: Probabilité, hs: effet hautement significatif, s: effet significatif, ns: effet non significatif. Les moyennes affectées de différentes lettres indiquent des différences significatives entre les traitements après le test de Student-Newman-Keuls $(\mathrm{P}<0,05)$, celles suivies de la même lettre ne sont pas significativement différentes $(\mathrm{P}>0,05)$ d'après le test.

\section{DISCUSSION}

Les résultats obtenus au cours des essais n'ont pas montré de discrimination variétale sous l'influence des différents ravageurs étudiés. Les résultats corroborent ceux obtenus par Dagoudo (2012), qui dans son étude sur l'identification des variétés adaptées à chaque zone du point de vue agro morphologique au Bénin, a trouvé que les variétés H 782-3, E 956-2 et H 769-5 ont été les meilleures.
Les résultats ont montré que l'augmentation des doses d'engrais n'est pas corrélée avec la pression parasitaire. Les trois traitements s'équivalent en ce qui concerne l'attaque des ravageurs. Cela pourrait s'expliquer par le fait qu'une augmentation de la dose d'engrais permet à la plante d'acquérir une certaine résistance aux ravageurs. Ces résultats sont similaires à ceux obtenus par Haougui (2005) qui a montré qu'une fertilisation adéquate est parfois nécessaire 
pour donner aux plantes un certain niveau de résistance aux ravageurs. Aussi nos résultats sont en harmonie avec ceux obtenus lors de la campagne 2010-2011 (CRA-CF, 2011). Par contre, nos résultats sont contraires à ceux trouvés par Luong et al. (2003) qui ont montré dans leurs études que la fertilisation azotée du riz améliore non seulement le développement des plants, mais aussi augmente la prolifération des insectes et maladies. Nos résultats sont également en discordance avec ceux trouvés par Luong et Heong (2005) qui ont montré que les parcelles du riz ayant reçu de l'engrais minéral ont hébergé plus de ravageurs par rapport à celles qui ont reçu de la fumure organique.

La sensibilité des variétés à la bactériose du cotonnier a aussi été évaluée. Les différentes observations faites ont montré que l'incidence de la bactériose a un effet significatif sur les variétés mises en comparaison à Angaradébou. En effet, la sévérité bactérienne est faible sur les variétés E 956-2 et H 782-3 et forte sur les H 279-1, I 875-3et H 769-5. Dans cette zone, il a été également constaté que les parcelles des variétés ayant reçu de fortes doses d'engrais ont exprimé moins la maladie que celles qui ont reçu de faibles doses. Nos résultats sont en harmonie avec ceux trouvés par Dagoudo (2012) qui ont révélé qu'au Nord, la pression bactérienne est forte sur la variété H279-1. Cependant, son étude n'a pas intégré les doses d'engrais. Nos résultats corroborent aussi ceux trouvés par Oluf et al. (1990) qui ont montré qu'un apport suffisant en potassium est nécessaire pour donner à la plante une certaine résistance aux maladies. De même, MAPAQ (2011) a montré qu'un apport adéquat d'engrais minéral confère à la plante une certaine résistance aux maladies.

\section{Conclusion}

Cette étude menée à Angaradébou au Bénin, a permis de comprendre que les ravageurs observés sur le coton n'ont pas de préférence variétale. En effet, ils parasitent les cotonniers sans distinction de variété. Il est aussi à noter que les variétés fertilisées avec de fortes doses d'engrais ont été moins infectées par la bactériose. Il convient cependant d'être prudent dans l'interprétation de tels résultats, quant à l'existence d'une relation de cause à effet entre l'application de fortes doses d'engrais et la sensibilité des variétés aux maladies qui n'est pas toujours vérifiée. Au vu des résultats obtenus et malgré l'influence des facteurs biotique et abiotique, la variété E 956-2 peut valablement remplacer la variété $H$ 279-1 actuellement en vulgarisation au nord du Bénin.

\section{REMERCIEMENTS}

Les auteurs remercient le gouvernement béninois pour avoir financé l'activité de recherche et tous ceux qui ont contribué à l'amélioration de ce document.

\section{REFERENCES}

Celini L. 2001. Le Puceron du cotonnier Aphisgossypii (Glover) et son parasite Aphelinus gossypii Timberlake en République centrafricaine. Insectes, 122(3): 7-10.

CRA-CF.2011. Rapport de campagne, 20102011, p.49

Dagoudo A. 2012. Caractérisation agromorphologique de huit variétés de Cotonnier (Gossypium hirsutum) dans les zones agro écologiques. Mémoire d'Ingénieur Agronome, Université de Parakou, Bénin, p.58

Dègla KP. 2012. Rentabilité économique et financière des exploitations cotonnières basées sur la Gestion Intégrée de la Fertilité des Sols et des Ravageurs au Nord-Bénin. Bulletin de la Recherche Agronomique du Bénin(BRAB), Numéro spécial: 26-35.

Estur G, Raymond G. 1988. Le coton dans le monde et en Afrique francophone de l'Ouest et du Centre. Coton et Fibres Tropicales, 43(3): 205-214.

Guy M. 2006. Contribution possibles des innovations génétiques pour l'amélioration de la compétitivité des files cotonnières africaines, p. 345. 
Haougui A. 2005. Les pratiques culturales et leurs effets sur les ennemis des cultures. NRAN, Niamey, p.10.

Hougni A. 2009. Qualités et valorisation du coton-fibre d'Afrique Zone Franc (AZF) dans les échanges internationaux. Thèse de doctorat ès sciences économiques, Université de Bourgogne, Bourgogne, p. 330.

Hussein K, Perret C, Hitimana L. 2005. Importance économique et sociale du coton en Afrique de l'Ouest : rôle du coton dans le développement, le commerce et les moyens d'existence. OCDE SAH/D 556, p. 71.

Kpadé PC. 2011. Adaptation de la coordination et nouvelles contradictions entre acteurs du système coton au Bénin face à la libéralisation économique. Thèse de Doctorat Unique, Université de Bourgogne, Bourgogne, p. 371.

Lançon J. 1996. Le cotonnier glandless: 350000 hectares en 1994. Agriculture et Développement, 9: 3-12.
Luong MC, Hoang DC, Phan TB, Luong TP, Jiaan C, Heong KL. 2003. Impacts of nutrition management on insect pests and diseases of rice. Omonrice, 11: 93-102.

Luong MC, Heong KL. 2005. Effects of organic fertilizers on insect pest and diseases of rice. Omonrice, 13: 26-33.

MAPAQ. 2011. Engrais et amendements minéraux. Ministère de l'Agriculture des Pêcheries et de l'Alimentation du Québec - Gouvernement du Québec.

Oluf CB, Ola K, Ole HL, Ian R. 1990. Agriculture et Fertilisation : les Engrais, leur Avenir. Norsk Hydro: Oslo ; 258 p.

Tchatchueng JB, Porte C. 2008. Specific Method for Spectrophotometric Determination of Gossypol. Asian Journal of Scientific Research, 1(6): 589597. 